\title{
Transcript changes in Vibrio cholerae in response to salt stress
}

\author{
Xiuping Fu' ${ }^{1,2}$, Weili Liang ${ }^{1,2}$, Pengcheng Du ${ }^{1,2}$, Meiying Yan ${ }^{1,2}$ and Biao Kan ${ }^{1,2^{*}}$
}

\begin{abstract}
Vibrio cholerae, which is a serious human intestinal pathogen, often resides and thrives in estuaries but requires major self-regulation to overcome intestinal hyperosmotic stress or high salt stress in water and food. In the present study, we selected multiple $\mathrm{O} 1$ and $\mathrm{O} 139$ group V. cholerae strains that were isolated from different regions and during different years to study their salt tolerance. Based on the mechanisms that other bacteria use to respond to high salt stress, we selected salt stress-response related genes to study the mechanisms which $V$. cholerae responds to high salt stress.

$V$. cholerae strains showed salt-resistance characteristics that varied in salt concentrations from $4 \%$ to $6 \%$. However, group $\mathrm{O} 1$ and group $\mathrm{O} 139$ showed no significant difference in the degree of salt tolerance. The primary responses of bacteria to salt stress, including $\mathrm{Na}^{+}$exclusion, $\mathrm{K}^{+}$uptake and glutamate biosynthesis, were observed in $\mathrm{V}$. cholerae strains. In addition, some sigma factors were up-regulated in $V$. cholerae strains, suggesting that $V$. cholerae may recruit common sigma factors to achieve an active salt stress response. However, some changes in gene transcript levels in response to salt stress in $V$. cholerae were strain-specific. In particular, hierarchical clustering of differentially expressed genes indicated that transcript levels of these genes were correlated with the degree of salt tolerance. Therefore, elevated transcript levels of some genes, including sigma factors and genes involved in peptidoglycan biosynthesis, may be due to the salt tolerance of strains. In addition, high salt-tolerant strains may recruit common as well as additional sigma factors to activate the salt stress response.
\end{abstract}

Keywords: Vibrio cholerae, Salt stress, Transcription, PCR array

\section{Introduction}

The ability to survive and thrive in severe environments is a prerequisite for pathogenic microorganisms to cause infectious diseases. Microorganisms must be able to adapt to environmental changes or stresses, such as temperature, $\mathrm{pH}$ and osmolarity pressure; for example, food-borne bacteria must adapt to high salt stress. The mechanisms that microorganisms use to respond to high salt stress have been investigated using model microorganisms such as Escherichia coli, Bacillus subtilis and Shewanella [1-5].

Generally, the mechanisms that bacteria use to respond to hypertonic stress include $\mathrm{Na}^{+}$ion exclusion and $\mathrm{K}^{+}$ion

\footnotetext{
* Correspondence: kanbiao@icdc.cn

'State Key Laboratory for Infectious Disease Prevention and Control, National Institute for Communicable Disease Control and Prevention, Chinese Center for Disease Control and Prevention, 155, Changbai Road, Changping, Beijing 102206, China

${ }^{2}$ Collaborative Innovation Center for Diagnosis and Treatment of Infectious Diseases, Hangzhou 310006, China
}

\section{() Biomed Central}

(c) 2014 Fu et al.; licensee Biomed Central. This is an Open Access article distributed under the terms of the Creative Commons Attribution License (http://creativecommons.org/licenses/by/4.0), which permits unrestricted use, distribution, and reproduction in any medium, provided the original work is properly credited. The Creative Commons Public Domain Dedication waiver (http://creativecommons.org/publicdomain/zero/1.0/) applies to the data made available in this article unless otherwise stated. uptake as well as the accumulation of compatible solutes, such as glutamate. Bacteria also recruit sigma factors that recognize specific promoter regions and that guide the assembly of RNA polymerase to regulate the expression of stress-response genes [6-9]. In addition to these common mechanisms, bacteria also use several unique mechanisms to adapt to high salt stress. For example, transcript levels of chemotaxis-related genes are upregulated in Desulfovibrio vulgaris Hildenborough, while those of chemotaxis-related genes are down-regulated or unaffected in Shewanella MR-1 $[4,10]$. In addition, critical enzymes involved in both aerobic and anaerobic respiration are significantly up-regulated in Shewanella MR-1 and D. vulgaris Hildenborough, while critical enzymes necessary for the tricarboxylic acid (TCA) cycle are down-regulated in Shewanella algae 2736 [4,10-12].

Vibrio cholerae, which is a serious human intestinal pathogen, often infects humans through contaminated water or food and will encounter many types of high salt

uns 
stress during its life cycle, including hyperosmotic stress in the intestine and high salt stress in water and food. Therefore, $V$. cholerae requires major self-regulation to overcome these stresses.

In this study, we examined changes in the expression of genes which have been suggested to be related to salt stress response, especially those involved in iron transport, amino acid biosynthesis and peptidoglycan biosynthesis, as well as sigma factors. We selected $8 \mathrm{~V}$. cholerae strains that were isolated from different regions and different years to study their salt tolerance and common mechanisms by which they respond to high salt stress. We analyzed transcript levels of salt stress-response related genes by using real-time PCR array.

\section{Materials and methods Strains}

$V$. cholerae strains used in this study were listed in Additional file 1: Table S1. All $8 \mathrm{~V}$. cholerae strains, including 4 serogroup O1 strains and 4 serogroup O139 strains, were toxigenic and carried $c t x A B$ cholera toxin genes. These strains were isolated from different sources, such as patients or water, and from different regions in China during different years (Additional file 1: Table S1).

\section{Growth conditions}

To identify the highest salt concentration that $V$. cholerae can tolerate, all $8 \mathrm{~V}$. cholerae strains were cultivated overnight in LB broth (1\% tryptone, $0.5 \%$ yeast extract) for use as seed cultures. Seed cultures were diluted at 1:100 and then cultivated at $37^{\circ} \mathrm{C}$ and $200 \mathrm{rpm}$ in triplicate in LB broth containing different amounts of $\mathrm{NaCl}(0.5 \%, 2 \%, 4 \%$, $5 \%$, or $6 \%$ ). Growth rates were measured spectrophotometrically $\left(\mathrm{OD}_{600}\right)$ once an hour.

\section{Sample preparation and total RNA extraction}

$V$. cholerae seed cultures were cultivated in triplicate in LB broth ( $1 \%$ tryptone, $0.5 \%$ yeast extract, $0.5 \% \mathrm{NaCl}$ ) for $5 \mathrm{~h}$ until reaching $\mathrm{OD}_{600}=1.0$. Next, $40 \mathrm{ml}$ of culture solution was harvested by centrifugation $\left(5500 \mathrm{rpm}\right.$ at $4^{\circ} \mathrm{C}$ for $10 \mathrm{~min}$ ) and resuspended twice in PBS. Then, cultures were cultivated in LB broth containing $0.5 \%$ (served as control sample) and $5 \% \mathrm{NaCl}$.

After $1 \mathrm{~h}$, total RNA was extracted using RNAiso reagent (TaKaRa), and genomic DNA was removed by incubation with DNase I (Ambion). Subsequently, the integrity of RNA was verified by analyzing intact ribosomal RNA bands using agarose gel electrophoresis, and the purity of RNA was determined using UV-spectrophotometry.

Each RNA sample $(1 \mu \mathrm{g})$ was reverse transcribed to cDNA using SuperScript III first-strand synthesis system (Invitrogen) according to the manufacturer's instructions. Biological triplicates cDNAs of each $V$. cholerae strain were mixed equally, and the mixture was used as a single qRT-PCR template.

\section{Real-time quantitative PCR (qRT-PCR)}

We selected 53 salt stress-response genes encoding sigma factor, glutamate biosynthesis, peptidoglycan biosynthesis and ion-transport proteins. Specific primers for each gene were designed using primer 3 batched run by a Perl script [13] and 109-168 bp products were amplified (see Additional file 2: Table S2 for primer details).

Fluorescence PCR amplifications were performed using SYBR Green EX Taq mix (TaKaRa) in a Bio-Rad CFX96 Real-Time PCR system. The relative transcript level of each gene (cultures cultivated in LB broth containing 5\% $\mathrm{NaCl})$ was determined by calculating $2^{-\Delta \Delta \mathrm{ct}}$ compared with the transcript level of control sample (cultures cultivated in $\mathrm{LB}$ broth containing $0.5 \% \mathrm{NaCl}$ ) using $\operatorname{gyrB}$ or thy $A$ gene as internal control. $\mathrm{ddH}_{2} \mathrm{O}$ was used as negative control for qRT-PCR. These genes were then used for GO term functional analysis and KEGG pathway analysis. Each experiment was conducted in triplicates.

\section{The internal control gene}

To identify the most stable internal control gene, 6 housekeeping genes were selected and evaluated using geNorm software. M values were calculated according to method mentioned in previous studies [14,15]. Primers for housekeeping genes amplification were designed according to previous study [16].

\section{Results}

Growth curves for $8 \mathrm{~V}$. cholerae strains under different $\mathrm{NaCl}$ concentrations

To determine the maximum salt concentration that $V$. cholerae strains can tolerate, 8 strains were cultivated in LB broth containing different concentrations of $\mathrm{NaCl}$ $(0.5 \%, 2 \%, 4 \%, 5 \%, 6 \%)$. Growth curves indicated that most of the strains could replicate in the presence of $0.5 \%$ to $5 \% \mathrm{NaCl}$. Strain VC3777 barely replicated in the presence of $5 \% \mathrm{NaCl}$; however, strain VC2035 replicated with a $\mathrm{NaCl}$ level of $6 \%$. Relative to the other strains, strains VC 3024 and VC2752 showed a noticeable growth delay before reaching log phase in the presence of $5 \%$ $\mathrm{NaCl}$. All of the $8 \mathrm{~V}$. cholerae strains required $\mathrm{NaCl}$ levels between $0.5 \%$ and $2 \%$ for optimal growth. When exposed to the highest salt concentration, all $8 \mathrm{~V}$. cholerae strains replicated slowly at first and entered stationary phase at a lower optical density (OD) than that when exposed to lower $\mathrm{NaCl}$ concentrations. Strain VC3777 barely replicated in $5 \% \mathrm{NaCl}$. Growth rates of strains VC3024 and VC2752 decreased significantly compared with those of the other strains in $5 \% \mathrm{NaCl}$. In addition, strain VC2035 replicated in $6 \% \mathrm{NaCl}$, while the other strains only replicated in the presence of $0.5 \%$ to $5 \% \mathrm{NaCl}$ (Figure 1). 


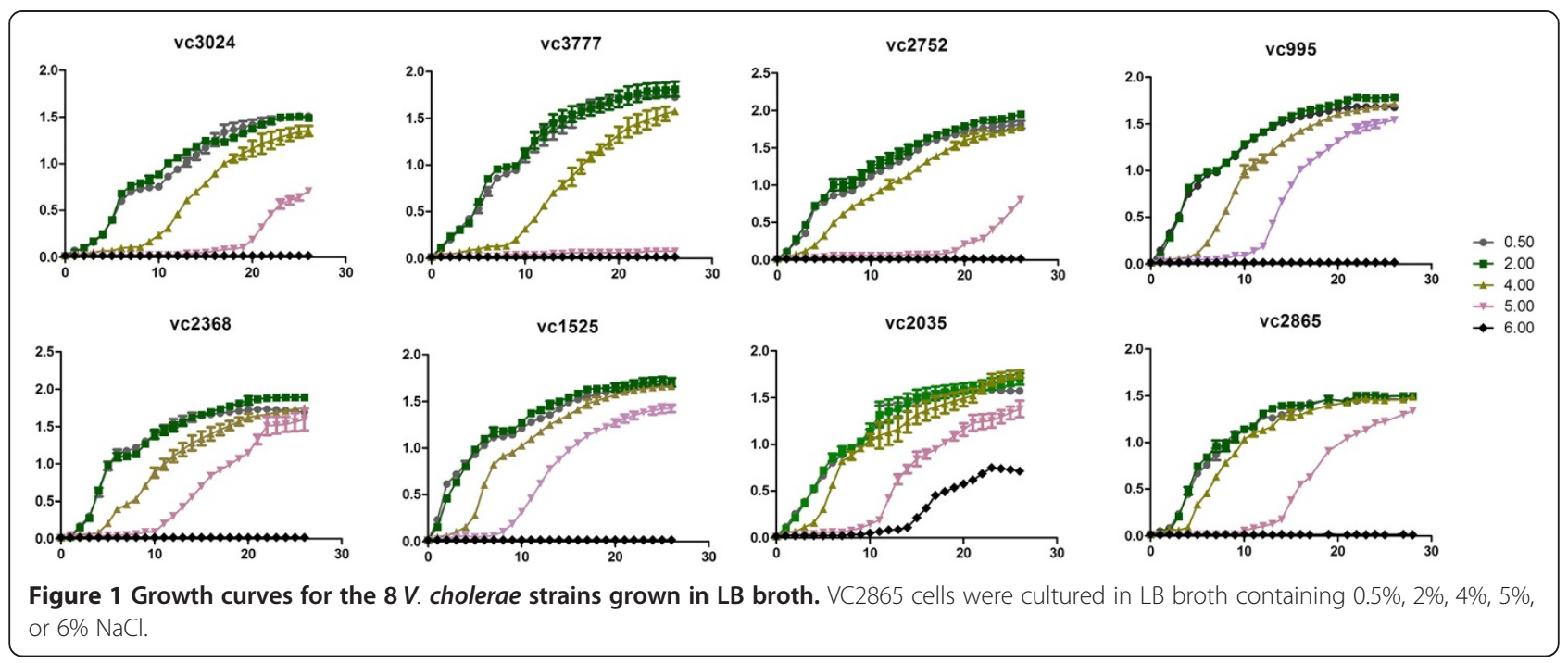

According to the degree of salt tolerance, three strains (VC3024, VC3777 and VC2752) were classified as the low salt-tolerant group, and five strains (VC2368,VC995, VC1525,VC2865,VC2035) were classified as the high salt-tolerant group. O1 and O139 showed no significant difference in the degree of salt tolerance. In our study, $5 \% \mathrm{NaCl}$ was selected to analyze salt stress responses of V. cholera.

\section{Stability evaluation of six candidate reference genes}

Relative gene transcript level should be normalized to that of a stable internal control gene. Therefore, in this study, we used gene stability measure $\mathrm{M}$ and geNorm algorithm to identify the most stable internal control gene of 6 selected housekeeping genes. All $M$ values of the 6 candidate genes were less than the cut-off value of 1.5 which is recommended by geNorm. The stability ranking of 6 candidate genes in $8 \mathrm{~V}$. cholerae strains was different. According to the result of $M$ values, $\operatorname{gyr} B$ $(\mathrm{M}<0.35)$ was the most stable gene for 7 of the $V$. cholerae strains, while thyA $(\mathrm{M}<0.05)$ was the most stable gene for strain VC1525. In addition, the mean Ct values of gyr $B$ and thy $A$ genes varied between 21.29 and 27.89, consistent with the recommended geNorm $C t$ values of $22-28$. In summary, we chose $\operatorname{gyr} B$ as the internal control gene for normalization of relative transcript levels for all of the $V$. cholerae strains except for strain VC1525, for which thyA was used as the internal control gene (Additional file 3: Table S3).

\section{Transcriptional changes in $V$. cholerae in response to salt stress}

We selected 53 genes, including sigma factor genes as well as genes involved in glutamate biosynthesis, iron transport and peptidoglycan biosynthesis. Total RNA was extracted using RNAiso reagent and then submitted to agarose gel electrophoresis. Clear bands of 16S rRNA and $23 \mathrm{~S}$ rRNA were observed. The OD260/OD280 ratios of RNA samples were between 1.8 and 2.0, indicating the high integrity and purity of RNA (Additional file 4: Figure S1). Relative transcript levels were normalized to the level of $\operatorname{gyr} B$ gene or thyA gene.

Transcript level changes of these 53 genes in response to salt stress were strain-specific but with some common characteristics (Additional file 5: Table S4). Genes encoding the large and small chains of glutamate synthase and those encoding the transporter system trkH (GL0042) were up-regulated in all 8 strains. Genes encoding the proton/glutamate symporter (gltP, GL1168 and GLA0088) and sodium/glutamate symporter ( $g l t S$, GLA0041) were down-regulated in most of the strains (6/8). Transcript levels of sigma factors were not the same in 8 strains. Three sigma factors (GL0330, regulator of sigma D; GL0706, sigma-54 modulation protein; GL2302, RNA polymerase sigma-70 factor) were up-regulated in all 8 strains, and some other sigma factors, such as GL0706 (sigma-54-modulation protein), GL1522 (sigma54-dependent response regulator), and GL1817 (sigma54-dependent transcriptional regulator) were up-regulated in most of the strains. Some sigma factors were upregulated in a portion of the strains but down-regulated in another portion of the strains, e.g., rpoS and $r p o H$ (GL0150 and GL0534). In addition, changes in transcript levels of genes involved in peptidoglycan synthesis were not identical in 8 strains.

Gene transcript profiles clustered by HCL (Hierarchical Clustering) showed that strains in the low salt-tolerant group (VC3024, VC3777 and VC2752) were clustered into one group, and strains in the high salt-tolerant group (VC2368, VC995, VC1525, VC2865, and VC2035) were 
clustered into another group (Figure 2). Transcript levels of these 53 genes were then analyzed using independent $t$-tests. As a result, transcript levels of 13 genes, including nine sigma factors (GL0665, GL2465, GL2466, GL2516, GL2530, GL0150, GL0534, GL1045 and GL2529) and four genes involved in peptidoglycan biosynthesis (GL0372, GL0870, GL0344 and GL2404), were significantly different between the high salt group and the low salt group. Moreover, transcript levels of these 13 genes in the high salt group were greater than those in the low salt group (Additional file 5: Table S4).

\section{Discussion}

In this study, we explored transcript level changes of salt-tolerant genes in $V$. cholerae exposed to elevated $\mathrm{NaCl}$ by using real-time PCR array. Eight $V$. cholerae strains, including 4 serogroup $\mathrm{O} 1$ strains and 4 serogroup O139 strains, were used in the present study.
These strains were isolated from different sources, such as patients, stool, and water (Additional file 1: Table S1). Our data showed no difference in the degree of salt tolerance between $\mathrm{O} 1$ group and $\mathrm{O} 139$ group.

Transcript levels were normalized to a stable internal control gene, which is necessary for qRT-PCR research. For each $V$. cholerae strain, 6 housekeeping genes were selected and evaluated using geNorm software. Housekeeping genes in $8 \mathrm{~V}$. cholerae strains showed different expression stability. gyr $B$ was the most stable gene for 7 strains, while thy $A$ was the most stable gene for $\mathrm{VC} 1525$ strain. GyrB or thyA, with the lowest $M$ value, was selected as the internal control gene for $V$. cholerae strains in our study. In addition, the Ct value of the internal control gene ranging from 21.29 to 27.89, was similar to those of target genes. In summary, the internal control gene selected by geNorm software was important for normalization and standardization. Thus,

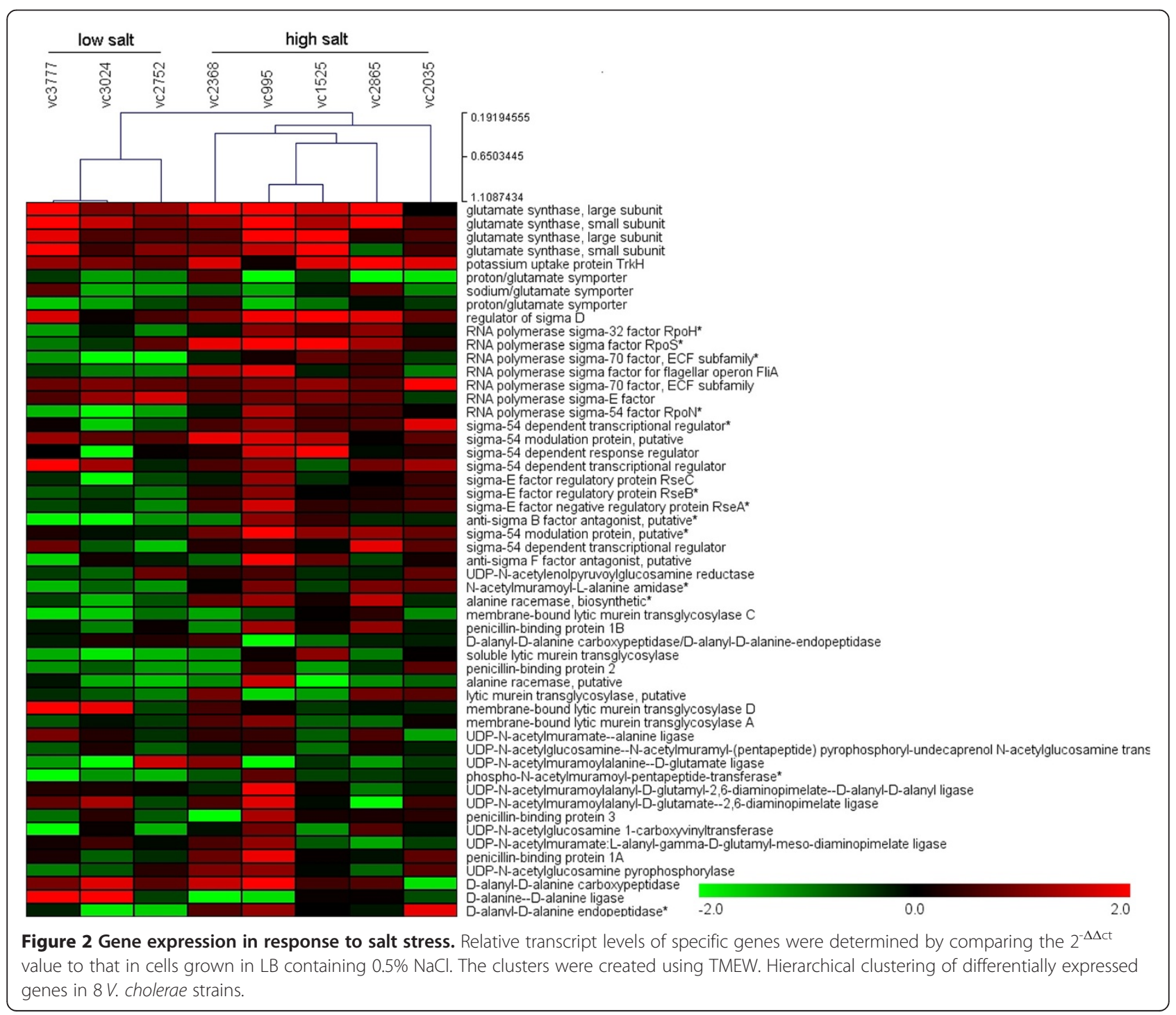


it is better to screen the internal control gene from 5-6 housekeeping genes for qRT-PCR array.

Eight $V$. cholerae strains in our study showed different salt-resistance characteristics, with the highest level of salt tolerance of $4 \%$ to $6 \% \mathrm{NaCl}$. However, no significant difference was observed in the degree of salt tolerance between group O1 and group O139. Changes in transcript levels of salt stress-response genes in $V$. cholera were strain-specific, indicating that the microbial stress response system is complicated.

Some common mechanisms were also found in the 8 strains of $V$. cholerae resisting to salt stress. The primary responses of bacteria to salt stress included $\mathrm{Na}^{+}$exclusion and $\mathrm{K}^{+}$uptake. Additionally, bacteria also accumulated glutamate to neutralize the increased number of cations [9]. In bacteria, three diverse $\mathrm{K}^{+}$transporter systems, Kup, $T r k$, and $K d p$, maintain the desired concentration of internal $\mathrm{K}^{+}$[17-19]. In our study, genes encoding the $\operatorname{trkH}$ transporter system were up-regulated in each of the 8 strains. However, genes encoding the symporters gltP and $g l t S$, responsible for simultaneously transferring one glutamate and one $\mathrm{H}^{+} / \mathrm{Na}^{+}$into the cell, were downregulated in all of the $V$. cholerae strains, which was consistent with the strategy used by other bacteria (such as E. coli) in response to salt stress [20]. These data indicated that $\mathrm{Na}^{+}$exclusion and $\mathrm{K}^{+}$uptake are the most important responses to high salt stress in $V$. cholerae. Genes encoding the large and small chains of glutamate synthase were up-regulated in all of the 8 strains, which indicated that glutamate biosynthesis is a primary mechanism used to counter salt stress in $V$. cholerae. In contrast, importing glutamate from the external environment is one of the primary mechanisms used to counter salt stress in S. algae [11]. In addition, three sigma factors, including the regulator of sigma $\mathrm{D}$, the sigma-54 modulation protein and the RNA polymerase sigma-70 factor, were up-regulated in all of the $V$. cholerae strains. Thus, these sigma factors are important for the response of $V$. cholerae to salt stress. Furthermore, the regulator of sigma D may up-regulate the expression of sigma-38 (such as rpoS) and of some stress-dependent genes by sequestering sigma-70 from their promoters $[21,22]$. In bacteria, the RNA polymerase sigma-70 factor plays important roles during various stress responses, especially in responses to osmotic pressure [23-26].

In our study, gene transcript profiles clustered by HCL showed that strains in the low salt-tolerant group were divided into one group and strains in the high salttolerant group were divided into another group, which suggested a correlation between gene transcript levels and the degree of salt tolerance. $t$-test analysis showed that transcript levels of 13 genes were significantly different between the high salt group and the low salt group. Moreover, transcript levels of the 13 genes in the high salt group were greater than those in the low salt group. We considered that the elevated transcript levels of these genes might be related to the high salt tolerance of strains. Transcript levels of sigma factors rpoS, rрoH and rpoN were up-regulated in high salt-resistant strains. Under normal growth conditions, the rpoS sigma factor is largely expressed in stationary phase, while under environmental stress, such as hyperosmosis, low $\mathrm{pH}$, and heat shock, the level and activity of rpoS increases rapidly, regulating approximately $10 \%$ of genome genes $[6,27,28]$. In E. coli, rpoH plays an important role during various stress responses, positively regulating more than 20 heat shock proteins when the bacterium encounters harsh environmental stress $[29,30]$.

It has been suggested that peptidoglycan layer thickening is not a common mechanism used by $V$. cholerae in response to salt stress. However, genes encoding peptidoglycan biosynthesis were variably regulated in different strains in response to salt stress. Specifically, four genes involved in peptidoglycan biosynthesis displayed greater transcript levels in the high salt group than those in the low salt group, suggesting that the elevated expression of these genes may improve the salt tolerance of strains. In a previous study, key genes involved in peptidoglycan biosynthesis were also shown to be up-regulated in S. algae [18].

In conclusion, $V$. cholerae use some common mechanisms to respond to high salt stress. For example, the trkH gene encoding $\mathrm{K}^{+}$transport protein, and genes encoding the large and small chains of glutamate synthase were up-regulated. In addition, $V$. cholerae may recruit some of sigma factors, such as the RNA polymerase sigma-70 factor, to activate the salt stress response pathway. Furthermore, strain-specific mechanisms also exist in $V$. cholerae, and transcript levels of some of these genes were correlated with the degree of salt tolerance. For example, transcript levels of the sigma factors rpoS and rpoH were elevated, indicating that the expression of these genes may improve the salt tolerance of strains. Thus, high salt-tolerant strains may recruit common sigma factors, as well as additional sigma factors, to active salt stress-response related genes.

\section{Additional files}

Additional file 1: Table S1. Background information of $V$. cholerae strains used in this study.

Additional file 2: Table S2. $q R T-P C R$ primers.

Additional file 3: Table S3. The most stable reference genes for $8 \mathrm{~V}$. cholerae strains under high salt condition.

Additional file 4: Figure S1. Analysis of RNA by agarose gel electrophoresis.

Additional file 5: Table S4. Raw data and complete list of up- and down-regulated genes. 


\section{Competing interests}

The authors declare that they have no competing interests.

\section{Authors' contributions}

XF carried out the molecular genetic studies, XF, WL, PD, MY and BK participated in the sequence alignment and drafted the manuscript. All authors read and approved the final manuscript.

\section{Funding}

This study was supported by the State Key Laboratory for Infectious Disease Prevention and Control of China [Grant number 2014SKLID101] and the Priority Project on Infectious Disease Control and Prevention [2012ZX10004215]. The funders had no role in study design, data collection and analysis, decision to publish, or preparation of the manuscript.

Received: 24 October 2014 Accepted: 13 December 2014

Published online: 30 December 2014

\section{References}

1. Boch J, Kempf B, Bremer E: Osmoregulation in Bacillus subtilis: synthesis of the osmoprotectant glycine betaine from exogenously provided choline. J Bacteriol 1994, 176:5364-5371.

2. Boch J, Kempf B, Schmid R, Bremer E: Synthesis of the osmoprotectant glycine betaine in Bacillus subtilis: characterization of the gbsAB genes. J Bacteriol 1996, 178:5121-5129.

3. Leblanc L, Leboeuf C, Leroi F, Hartke A, Auffray Y: Comparison between $\mathrm{NaCl}$ tolerance response and acclimation to cold temperature in Shewanella putrefaciens. Curr Microbiol 2003, 46:157-162.

4. Liu Y, Gao W, Wang Y, Wu L, Liu X, Yan T, Alm E, Arkin A, Thompson DK, Fields MW, Zhou J: Transcriptome analysis of Shewanella oneidensis MR-1 in response to elevated salt conditions. J Bacterio/ 2005, 187:2501-2507.

5. von Blohn C, Kempf B, Kappes RM, Bremer E: Osmostress response in Bacillus subtilis: characterization of a proline uptake system (OpuE) regulated by high osmolarity and the alternative transcription factor sigma B. Mol Microbiol 1997, 25:175-187.

6. Hengge-Aronis R: Recent insights into the general stress response regulatory network in Escherichia coli. J Mol Microbiol Biotechnol 2002, 4:341-346.

7. Hughes KT, Mathee K: The anti-sigma factors. Annu Rev Microbiol 1998, 52:231-286

8. Roesser M, Muller V: Osmoadaptation in bacteria and archaea: common principles and differences. Environ Microbio/ 2001, 3:743-754.

9. Sleator RD, Hill C: Bacterial osmoadaptation: the role of osmolytes in bacterial stress and virulence. FEMS Microbiol Rev 2002, 26:49-71.

10. Mukhopadhyay A, He Z, Alm EJ, Arkin AP, Baidoo EE, Borglin SC, Chen W, Hazen TC, He Q, Holman HY, Huang K, Huang R, Joyner DC, Katz N, Keller M, Oeller P, Redding A, Sun J, Wall J, Wei J, Yang Z, Yen HC, Zhou J, Keasling JD: Salt stress in Desulfovibrio vulgaris Hildenborough: an integrated genomics approach. J Bacteriol 2006, 188(11):4068-4078.

11. Fu X, Wang D, Yin X, Du P, Kan B: Time course transcriptome changes in Shewanella algae in response to salt stress. PLoS One 2014, 9:e96001.

12. He Z, Zhou A, Baidoo E, He Q, Joachimiak MP, Benke P, Phan R, Mukhopadhyay A, Hemme CL, Huang K, Alm EJ, Fields MW, Wall J, Stahl D, Hazen TC, Keasling JD, Arkin AP, Zhou J: Global transcriptional, physiological, and metabolite analyses of the responses of Desulfovibrio vulgaris hildenborough to salt adaptation. App/ Environ Microbiol 2010, 76(5):1574-1586.

13. Lincoln S. E. DME, Lander E. S: Primer: a computer program for automatically selecting PCR primers. 1991. Whitehead Institute Technical Report

14. Mestdagh $P$, Van Vlierberghe $P$, De Weer A, Muth D, Westermann F, Speleman F, Vandesompele J: A novel and universal method for microRNA RT-qPCR data normalization. Genome Biol 2009, 10:R64.

15. Vandesompele J, De Preter K, Pattyn F, Poppe B, Van Roy N, De Paepe A, Speleman F: Accurate normalization of real-time quantitative RT-PCR data by geometric averaging of multiple internal control genes. Genome Biol 2002, 3:RESEARCH0034.

16. Zhang Cuicai PB, Jiang $X$, Kan B: Selection of reference genes for gene expression analysis in V. cholera. Chin J Zoonoses 2014, 30:6.
17. Bakker EP: Cell K+ and K+ transport systems in prokaryotes. In Alkali Cation Transport Systems in Prokaryotes. Edited by Bakker EP. CRC Press; 1993, pp. 205-224

18. Epstein W: Kdp, a bacterial P-type ATPase whose expression and activity are regulated by turgor pressure. Acta Physiol Scand Suppl 1992, 607:193-199.

19. Schlosser A, Meldorf M, Stumpe S, Bakker EP, Epstein W: TrkH and its homolog, TrkG, determine the specificity and kinetics of cation transport by the Trk system of Escherichia coli. J Bacteriol 1995, 177:1908-1910.

20. Tolner B, Ubbink-Kok T, Poolman B, Konings WN: Characterization of the proton/glutamate symport protein of Bacillus subtilis and its functional expression in Escherichia coli. J Bacteriol 1995, 177:2863-2869.

21. Mitchell JE, Oshima T, Piper SE, Webster CL, Westblade LF, Karimova G, Ladant D, Kolb A, Hobman JL, Busby SJ, Lee DJ: The Escherichia coli regulator of sigma 70 protein, Rsd, can up-regulate some stressdependent promoters by sequestering sigma 70. J Bacteriol 2007, 189:3489-3495.

22. Jishage $M$, Ishihama $A$ : Transcriptional organization and in vivo role of the Escherichia coli rsd gene, encoding the regulator of RNA polymerase sigma D. J Bacteriol 1999, 181:3768-3776.

23. Abromaitis S, Koehler JE: The Bartonella quintana extracytoplasmic function sigma factor RpoE has a role in bacterial adaptation to the arthropod vector environment. J Bacterio/ 2013, 195:2662-2674.

24. Alvarez-Martinez CE, Lourenco RF, Baldini RL, Laub MT, Gomes SL: The ECF sigma factor sigma( $\mathrm{T})$ is involved in osmotic and oxidative stress responses in Caulobacter crescentus. Mol Microbiol 2007, 66:1240-1255.

25. Helmann JD: The extracytoplasmic function (ECF) sigma factors. Adv Microb Physiol 2002, 46:47-110.

26. Tettmann B, Dotsch A, Armant O, Fjell CD, Overhage J: Knockout of extracytoplasmic function sigma factor ECF-10 affects stress resistance and biofilm formation in Pseudomonas putida KT2440. Appl Environ Microbiol 2014, 80:4911-4919.

27. Arsene F, Tomoyasu T, Bukau B: The heat shock response of Escherichia coli. Int J Food Microbiol 2000, 55:3-9.

28. Hengge R: Proteolysis of sigmaS (RpoS) and the general stress response in Escherichia coli. Res Microbiol 2009, 160:667-676.

29. Dong T, Schellhorn HE: Control of RpoS in global gene expression of Escherichia coli in minimal media. Mol Genet Genomics 2009, 281:19-33.

30. Heitzer A, Mason CA, Hamer G: Heat shock gene expression in continuous cultures of Escherichia coli. J Biotechnol 1992, 22:153-169.

\section{doi:10.1186/s13099-014-0047-8}

Cite this article as: Fu et al.: Transcript changes in Vibrio cholerae in response to salt stress. Gut Pathogens 2014 6:47

\section{Submit your next manuscript to BioMed Central and take full advantage of:}

- Convenient online submission

- Thorough peer review

- No space constraints or color figure charges

- Immediate publication on acceptance

- Inclusion in PubMed, CAS, Scopus and Google Scholar

- Research which is freely available for redistribution

Submit your manuscript at www.biomedcentral.com/submit
C BioMed Central 